\title{
Cambio en la distribución de goodeidos (Osteichthyes: Cyprinodontiformes: Goodeidae) en cuencas hidrológicas del centro de México
}

\author{
Changes in the distribution of goodeids (Osteichthyes: Cyprinodontiformes: Goodeidae) in \\ river basins of Central Mexico
}

Omar Domínguez-Domínguez ${ }^{1,3 *}$, Luis Zambrano², Luis Humberto Escalera-Vázquez ${ }^{1,4}$, Rodolfo Pérez-
Rodríguez ${ }^{1,4}$ y Gerardo Pérez-Ponce de León ${ }^{2}$
'Laboratorio de Biología Acuática, Facultad de Biología, Universidad Michoacana de San Nicolás de Hidalgo, Edificio R, planta baja, Ciudad
Universitaria, 58000, Morelia, Michoacán, México.
${ }^{2}$ Departamento de Zoología-Instituto de Biología, Universidad Nacional Autónoma de México. Apartado postal 70-153, 04510 México, D.F., México.
${ }^{3}$ Posgrado en Ciencias del Mar y Limnología, CMyL, Universidad Nacional Autónoma de México, Apartado postal 70-305, 04510 México, D. F.,
México.
${ }^{4}$ Posgrado en Ciencias Biológicas-Instituto de Biología, Universidad Nacional Autónoma de México. Apartado postal 70-153, 04510 México, D.F.,
México.
*Correspondencia: odoming@jupiter.umich.mx

Resumen. La Mesa Central de México cuenta aproximadamente con 100 especies nativas de peces, el 70\% son endémicas de la región. Los ecosistemas de esa área geográfica son sobre los que mayor impacto ha tenido la actividad antropogénica. El grupo de peces más representativo es el de los goodeidos que aparentemente han sobrevivido a grandes cambios de calidad de agua y flujo hídrico. Para entender los cambios en la distribución de esta familia, el presente trabajo documenta las desapariciones y extinciones locales de especies de goodeidos y su relación con las diferentes regiones de la Mesa Central. Para conocer su distribución previa, se realizó una búsqueda bibliográfica y en bases de datos de colecciones. Estos registros se compararon con recolectas realizadas entre junio de 1999 y septiembre del 2004 en 287 localidades en 15 estados de la República Mexicana. Se cubrió el 95\% de los sitios de recolecta que presentaban registros previos. La comparación entre distribuciones arrojó que en el 68\% de estos sitios se registró la desaparición de alguna especie. En 9 de las 18 regiones, las especies previamente registradas redujeron su distribución a la mitad, han desaparecido 5 en más del $50 \%$ de su intervalo de distribución y al menos 2 se señalan como extintas. Las especies se clasificaron según su vulnerabilidad y las localidades fueron categorizadas de acuerdo al promedio de desaparición de poblaciones. Se discute el efecto de las extinciones sobre la viabilidad de las especies a largo plazo, y la pertinencia de establecer áreas de conservación para los goodeidos. Las extinciones locales pueden afectar la viabilidad de las especies, y por lo tanto es urgente generar áreas de conservación para los godeidos.

Palabras clave: goodeidos, extinciones locales, vulnerabilidad de especies, peces dulceacuícolas, conservación.

\begin{abstract}
The Mexican central plateau hosts around 100 native freshwater fish species, $70 \%$ of which are endemic to the region. Freshwater ecosystems in this area are highly impacted by anthropogenic activities. Goodeids are the most representative fish in lakes and rivers in the central plateau. In recent decades, these fishes have suffered from water quality depletion and modification of hydrological regimes, leading to reduction of their original distributions. To understand changes in the distribution of species of this family, we documented local extinctions of its species and its relation with their sensitivity to environmental changes. Previous distribution records for each species were obtained from bibliographical sources and museum collection data. We compared previous distribution with more recent fieldwork data, which was conducted between 1999 and 2004, in 287 localities in 15 states of the country. Fieldwork covered 95\% of the localities sampled in previous records. We found local extinction in 68\% of the localities surveyed. In 9 of the 18 regions, species distribution was reduced to half of their reported areas. Five species have disappeared in 50\% of their original distributional range, and at least 2 species are already extinct. We classified Goodeid species according to their vulnerability and localities were categorized by the quantity of local extinctions. Local extinctions may affect species viability, and it is therefore urgent to generate areas of conservation for goodeids.
\end{abstract}

Key words: Goodeids, local extinctions, species vulnerability, freshwater fishes, fish conservation.

Recibido: 27 septiembre 2007; aceptado: 19 mayo 2008 


\section{Introducción}

Los ecosistemas acuáticos de la Mesa Central de México (sensu Barbour, 1973; Echelle y Echelle, 1984) están sujetos a un fuerte impacto por la actividad antropogénica. Esta región cuenta con el $20 \%$ del agua superficial del país (Athie, 1987) pero alberga el $73 \%$ de la población humana y el $67 \%$ de la actividad industrial (Cotler et al., 2006). Algunos de los factores que contribuyen a su acelerado deterioro son: la contaminación de las aguas, la disminución de los mantos acuíferos, la deforestación de las cuencas, la desecación intencional de cuerpos de agua, la introducción de especies exóticas y sus parásitos, y una sobre explotación pesquera (Lyons et al., 1998; Zambrano et al., 1999; Mercado-Silva et al., 2002; Contreras-Balderas et al., 2003; Domínguez-Domínguez et al., 2005b).

Aún cuando existe un gran impacto antropogénico, la Mesa Central de México cuenta con una extraordinaria diversidad íctica de aproximadamente 100 especies nativas, el $70 \%$ de las cuales son endémicas de la región (Miller et al., 2005). Dentro de los grupos de peces más representativos se encuentra la subfamilia Goodeinae, que está conformada por 19 géneros y 41 especies. La distribución de las especies de esta subfamilia se extiende de la cuenca del ríos Mezquital y Salado (en el norte), hasta la del río Balsas por la vertiente del Pacífico y la del río Pánuco por la del golfo de México hacia el sur, abarcando los estados de Durango, Zacatecas, Nayarit, San Luis Potosí, Jalisco, Colima, Guanajuato, Aguascalientes, Querétaro, Hidalgo, Tlaxcala, México, Morelos, Michoacán, Puebla, Guerrero y Distrito Federal.

Los goodeidos son un grupo de peces cuya conservación está en riesgo puesto que muchas de sus especies se encuentran en peligro de extinción (Duncan y Lockwood, 2001; Domínguez-Domínguez et al., 2005a). Algunas especies de esta subfamilia están registradas como extintas (v.gr. Characodon garmani y Ameca splendens) por la Unión Internacional para la Conservación de la Naturaleza (UICN); otras 2, Skiffia francesae y Zoogoneticus tequila las registra como extintas la Norma Oficial Mexicana NOM-059 (DOF, 2001), pero con poblaciones en cautiverio. La segunda de estas especies cuenta con registros recientes de poblaciones en la naturaleza (De la Vega-Salazar et al., 2003); Hay especies que no se ha logrado recolectar desde hace más de 5 años (Allotoca goslinae y Allodontichthys polylepis). Aunado a lo anterior, la NOM-059 registra otras 8 en peligro y 4 amenazadas; por lo tanto, cerca del $30 \%$ de esta subfamilia está en alguna categoría de riesgo. Sin embargo, evaluaciones recientes sugieren un panorama más pesimista, aumentando al $60 \%$ las especies en peligro o amenazadas (DomínguezDomínguez et al., 2005b).
Los peces tienen diferente capacidad para responder al deterioro del hábitat (De la Vega-Salazar et al., 2003). Por ejemplo, el tamaño de su descendencia puede ser un factor que contribuye a la vulnerabilidad. Especies que producen poca descendencia son más susceptibles a la extinción, ya que tienen una limitada capacidad de dispersión y recolonización (Efford et al., 1997). Otros factores relacionados con la vulnerabilidad de las especies están asociados con la resistencia a cambios drásticos en los niveles de perturbación del ambiente donde habitan (Matthews y Marsh-Matthews, 2003; Lytle y LeRoy, 2004), la especificidad en la alimentación (Weis et al., 1999) o la capacidad competitiva con especies exóticas (Maezono y Miyashita, 2003; Escalera-Vázquez, 2006). Por lo tanto, el considerar la adecuación de las especies para responder a cambios en el hábitat puede ser fundamental para hacer predicciones más precisas de extinciones potenciales y para generar planes de conservación a nivel regional.

Un estudio reciente basado en la distribución potencial de los goodeidos sugiere que paradójicamente, las áreas donde es probable encontrar un mayor número de especies son también aquellas que han sido transformadas de manera drástica por la actividad humana (Domínguez-Domínguez et al., 2006a). Sin embargo, este estudio no consideró la susceptibilidad de las diferentes especies a los cambios generados por el hombre.

El presente trabajo tiene como objetivo utilizar la información derivada de un intenso trabajo de campo de 5 años para comparar la distribución actual de las poblaciones de peces de la subfamilia Goodeinae con los registros previos existentes, con lo que se podrá documentar la desaparición y extinciones locales ocurridas durante ese periodo. También, el trabajo pretende relacionar estas desapariciones con la sensibilidad de las especies a los cambios en el ambiente.

\section{Materiales y métodos}

Para realizar el análisis de la distribución de las especies de goodeidos en el centro de México se realizó, en primera instancia, una extensa recopilación bibliográfica y de información contenida en las bases de datos de 14 colecciones ictiológicas públicas y privadas, de 6 países (Cuadro 1). Los datos de colecciones sólo fueron tomados en cuenta cuando fue posible corroborar la correcta identificación del material por los autores del presente trabajo, o bien, por algún otro especialista reconocido. Estos datos comprenden el periodo de 1930 a 1998. Al conjunto de datos obtenidos de esta recopilación en lo sucesivo se le llamará "registros previos".

Para obtener información sobre la distribución actual 
Cuadro 1. Bases de datos de colecciones científicas y otras organizaciones de las cuales se obtuvo información para el análisis histórico de los goodeidos que habitan en las cuencas del centro de México

\begin{tabular}{lc}
\hline Colección & País \\
\hline Colección de Peces de la Universidad Michoacana & México \\
Colección Nacional de Peces del Instituto de Biología de la UNAM & México \\
Colección Nacional de Peces Dulceacuícolas del Instituto Politécnico Nacional & México \\
Colección de Peces de la Universidad Autónoma de Nuevo León & México \\
Colección Regional de Peces de la Universidad Autónoma de Querétaro & México \\
Colección Regional de Peces de la Universidad Autónoma del Estado de Morelos & México \\
Colección de Peces del Museo Nacional de Ciencias Naturales de Madrid & España \\
Colección de Peces del Museo de Zoología de la Universidad de Michigan & Estados Unidos \\
Asociacion Americana de Peces Vivíparos & Estados Unidos \\
Colección de Peces de la Universidad de Tulane & Estados Unidos \\
Colección de Peces del grupo POECILIA & Holanda \\
Colección de Peces del grupo DGLZ & Alemania \\
Asociación Británica de Peces Vivíparos & Reino Unido \\
Grupo Internacional para el estudio de los goodeidos & Varios \\
\hline
\end{tabular}

de los goodeidos y contrastarla con los registros previos, se realizaron 47 salidas al campo, en 287 localidades, que representan el $95 \%$ de las localidades con registros previos. Todas las localidades muestreadas estuvieron comprendidas en cuerpos de agua lóticos y lénticos dentro de la Mesa Central de México, abarcando los estados de Durango, Zacatecas, Aguascalientes, Nayarit, San Luis Potosí, Querétaro, Jalisco, Estado de México, Guanajuato, Morelos, Puebla, Michoacán, Hidalgo, Colima y Guerrero. Estas localidades fueron agrupadas en las 18 regiones identificadas por Domínguez-Domínguez et al. (2006b) (Fig. 1). Las recolectas se realizaron entre junio de 1999 y septiembre del 2004.

Los métodos de recolecta se basaron en diferentes artes de pesca con el fin de capturar el mayor número de especies posible: chinchorro, atarraya, trampas tipo naza, electropesca y redes de mano. El empleo de cada una de las artes de pesca dependió del tipo de ambiente, eligiendo los métodos que generaran el menor impacto ambiental y mayor captura.

La mayoría de los organismos fueron identificados en campo y regresados al medio de donde fueron extraídos. Una muestra representativa de los organismos recolectados se preservó en alcohol al $70 \%$ o en formol al $10 \%$ para corroborar su identificación con base en las claves de Álvarez del Villar (1970) y Miller et al. (2005). Los organismos recolectados fueron depositados en la Colección de Peces de la Universidad Michoacana (CPUM), en la Colección Nacional de Peces del Instituto de Biología de la UNAM (CNPE) y en la Colección Regional de Peces de la Universidad Autónoma de Querétaro. Algunos más fueron transportados vivos al laboratorio e incorporados al "Programa de conservación y manejo de goodeidos mexicanos" del Laboratorio de Biología Acuática de la Universidad Michoacana de San Nicolás de Hidalgo. El arreglo taxonómico aquí presentado está basado en lo expuesto en Doadrio y Domínguez (2004). En el proceso de identificación y revisión taxonómica se observaron cambios en la nomenclatura de algunas especies en determinadas cuencas (v. gr. presencia de Xenotoca variata en la cuenca del río Ameca). En estos casos se realizó una revisión de la historia en la taxonomía y nomenclatura de la especie en cuestión. En aquellos casos que se tuvo acceso a los organismos recolectados originalmente, se realizó una revisión taxonómica y de nomenclatura de la especie.

Las 287 localidades donde se recolectaron organismos fueron dividas para su análisis en 3 categorías de acuerdo con: 1) donde se recolectó la totalidad de las especies encontradas en registros previos; 2) donde se recolectó al menos una de las especies encontradas en registros previos, pero no su totalidad, y 3) donde no se recolectó ninguna de las especies encontradas en registros previos. Con la información obtenida de la categorización de las localidades y basados en las coordenadas geográficas se elaboró un mapa en el programa ArcView 3.2. (Fig. 1). En este mapa se representan las 3 categorías en que se dividieron las localidades.

Las especies de goodeidos fueron clasificadas con base en su vulnerabilidad (en sensibles, moderadamente 


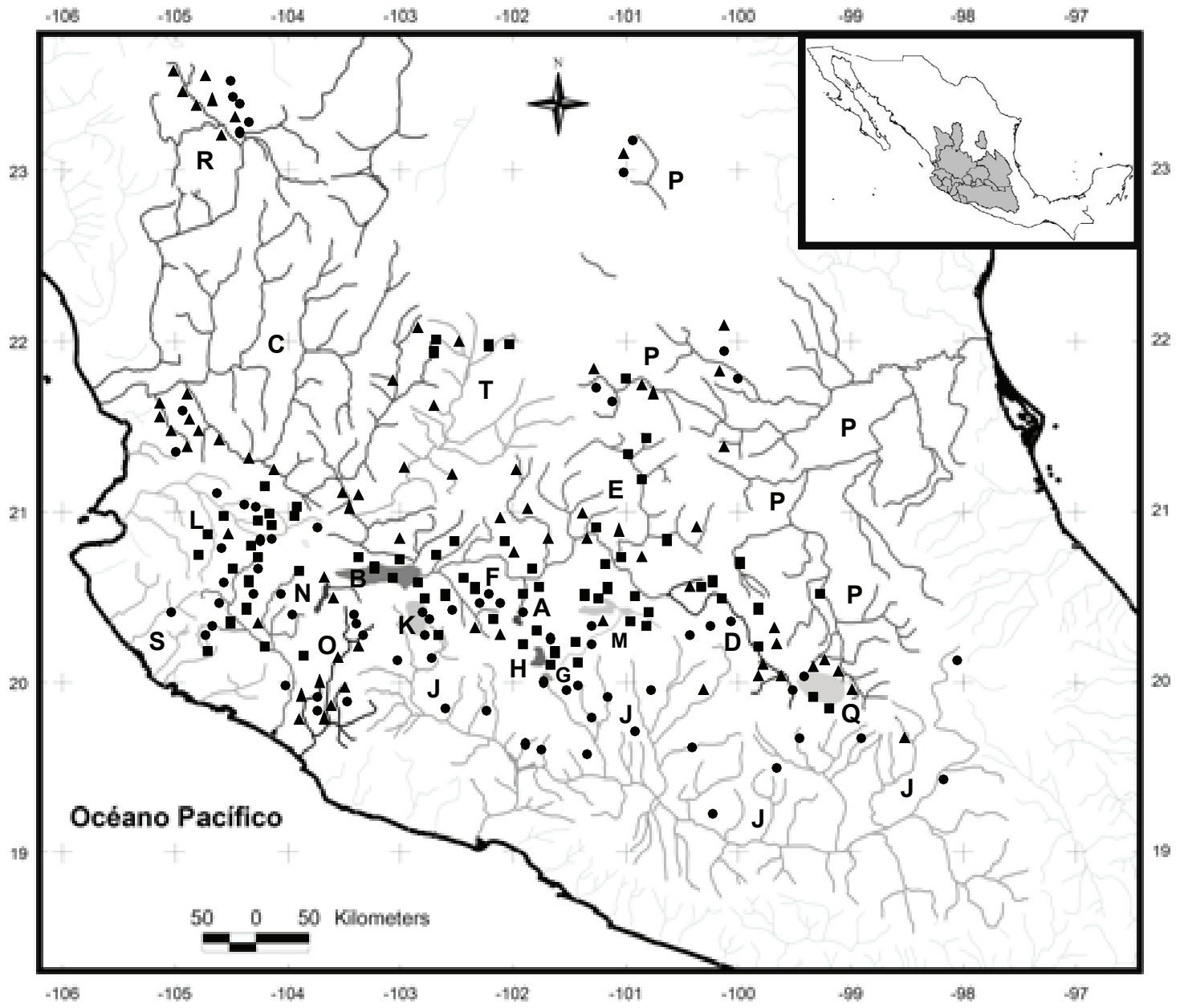

Figura 1. Localidades en las 18 regiones biogeográficas del centro de México donde se recolectaron especies de goodeidos con registros previos: - localidades que contaban con registros; - localidades donde alguna de las especies que contaba con registros previos no fue recolectada; $\boldsymbol{\Delta}$ localidades con registros previos donde no se recolectó ninguna especie. Cada región está indicada con una tonalidad diferente y una letra. $\mathrm{A}=\mathrm{Zacapu}, \mathrm{B}=$ Chapala, $\mathrm{C}=$ Santiago, $\mathrm{D}=$ Alto Lerma, $\mathrm{E}=\mathrm{Medio}$ Lerma, $\mathrm{F}=\mathrm{Bajo} \mathrm{Lerma}, \mathrm{G}=$ Zirahuén, $\mathrm{H}=$ Pátzcuaro, $\mathrm{J}=$ Balsas, $\mathrm{K}=$ Cotija, $\mathrm{L}=$ Ameca, $\mathrm{M}=$ Cuitzeo, $\mathrm{N}=$ Armería-Ayuquila, $\mathrm{O}=\mathrm{C}$ Coahuyana-Tamazula, $\mathrm{P}=\mathrm{Pánuco}$, $\mathrm{Q}=$ Valle de México, $\mathrm{R}=$ Mezquital, $\mathrm{S}=$ Purificación-Marabasco, T= Verde. Un mismo símbolo puede identificar 2 localidades muy cercanas; algunos llegan a sobreponerse.

sensibles y tolerantes) con ayuda de los trabajos de Lyons et al. (2000) y Mercado-Silva et al. (2002). En estos trabajos se definen como especies sensibles aquellas restringidas a sistemas prístinos; moderadamente sensibles las que se pueden encontrar en lugares poco perturbados, y tolerantes las que se encuentran con salud poblacional en sistemas contaminados, turbios y relativamente anóxicos (Ledesma-Ayala, 1987). Con los registros previos y de campo se construyó una matriz de datos de donde se obtuvo la tasa de permanencia para cada especie (TP) en cada una de las regiones donde naturalmente se distribuye. La TP se obtuvo de la siguiente manera:

$$
T P=\frac{R A}{R P}
$$

donde RA = número de localidades con registros actuales de cada región mientras que $\mathrm{RP}=$ número de localidades con registros previos de cada región. Así, una TP $=1$ sugiere que en la actualidad la especie se encuentra en todos los sistemas donde se registró previamente, mientras que una $\mathrm{TP}=0.5$ sugiere que la especie sólo se encontró en la mitad de los sitios donde originalmente fue registrada. La TP se usó para comparar la reducción de hábitat de las especies sensibles, moderadamente sensibles y tolerantes. También se usó para obtener el valor de presencia por especie. Para obtener este valor se obtuvo el promedio de TP para cada especie en todas las regiones donde ha estado presente.

Para comparar las regiones donde desaparecieron más 
especies con aquellas donde su permanencia fue mayor, se agruparon en tres categorías: 1) regiones con especies que en promedio tuvieron una TP igual o menor al 33\%; 2) regiones con especies con una TP promedio entre el 34 y el $66 \%$, y 3) regiones en las que la permanencia ha sido mayor al 66\%. Posteriormente se comparó el número de especies endémicas y extintas en los 3 grupos.

Las regiones se agruparon con base en la proporción de especies tolerantes, moderadamente tolerantes y sensibles que habitan cada localidad y se utilizó la prueba de análisis de varianza para distinguir si hay diferencia en los valores de TP de estos 3 grupos. Para los datos que no cumplieron con la prueba de normalidad se realizó una prueba de KruskalWallis (Siegel y Castellan, 1988). En los resultados de las pruebas que fueron significativas se realizó el análisis de post-hoc de Tukey (para las paramétricas) y Dunn (para las no paramétricas). Las localidades se visitaron al menos 3 veces, salvo en raras ocasiones (ej. localidades donde el cuerpo de agua desapareció o se desecó, o cuerpos de agua altamente contaminados). Para los análisis estadísticos sólo se tomaron en cuenta a las que se les hicieron no menos de 3 visitas durante los periodos de lluvias y secas en el lapso comprendido entre 1999 y 2004.

\section{Resultados}

Peces. Con base en las salidas al campo se registraron 38 de las 41 especies de goodeidos reconocidas (sensu Doadrio y Domínguez, 2004; Domínguez-Domínguez et al., 2005b). En 80 localidades (32\% del total muestreado) se recolectaron todos los organismos mencionados en registros previos; en 89 (36\%) se registró la extinción local de al menos una de las especies obtenidas en registros previos; mientras que en 78 (32\%) no se colectó ninguna de las especies en registros previos (Cuadro 2 y Fig. 1).

Las extinciones locales de especies por región ascienden a un total de 20. Alloophorus robustus es la especie que ha desaparecido en un mayor número de regiones (4), representando el $40 \%$ de las regiones donde esta especie se distribuía anteriormente. Xenotoca melanosoma ha desaparecido en 3 localidades, representando una disminución del $75 \%$ de su distribución previa por región (Cuadro 3). A pesar de la intensa búsqueda, no pudieron recolectarse Characodon garmani, Skiffia francesae y Zoogoneticus tequila. La primera es microendémica de una porción de la región de Parras, Coahuila y las últimas de la región de Teuchitlán, Jalisco, en la cuenca del río Ameca.

En cuanto a la tasa de permanencia de las especies respecto a su vulnerabilidad, las tolerantes tuvieron una TP significativamente mayor a las sensibles y moderadas
$(\mathrm{F}=15.42 ; \mathrm{p}<0.001)$ (Fig. 2). Las especies sensibles desaparecieron en un $70 \%$ de las localidades donde se obtuvieron en registros previos, mientras que las tolerantes únicamente se redujeron aproximadamente en un $25 \%$.

Localidades. Las regiones con mayor número de localidades en las que ninguna de las especies de los registros previos fueron recolectadas en nuestros muestreos son: Santiago con 20 (representando el $80 \%$ de sus localidades muestreadas) y el Lerma medio con 9 (52\%). Las regiones con el mayor número de localidades en las que al menos una de las especies con registros previos no fue recolectada son: Ameca con 13 (representando el $52 \%$ de sus localidades muestreadas), seguido de ArmeríaAyuquila con 10 (50\%). En el $100 \%$ de las localidades muestreadas en la zona de Chapala, Zirahuén y Lerma medio, se registró la extinción de al menos una de las especies registradas previamente, seguidas de Santiago con un $94 \%$. La región del río Balsas, una de las que cuenta con la menor diversidad de goodeidos, es la que presenta un menor porcentaje de extinciones locales por sitio de recolecta $(8 \%)$.

A partir del promedio de la tasa de permanencia de cada especie (Cuadro 3), se conformaron los grupos de las regiones donde más han desaparecido los goodeidos (Cuadro 4). El número de especies endémicas en cada grupo de regiones no varía significativamente, aun cuando la tendencia sugiere que el grupo de regiones con mayor número de desapariciones (grupo 1) cuenta con menos especies endémicas en promedio (Fig. 3). Al comparar el número de especies extintas localmente, se presentó una diferencia significativa en la cantidad de especies extintas en el grupo de regiones con menor promedio en la TP de las especies (grupo 1). El grupo con mayor promedio en la TP de sus especies no cuenta con una sola extinción local (Fig. 3).

No se encontraron diferencias significativas al comparar el tipo de región con el número de especies sensibles, moderadas y tolerantes respectivamente (Fig. 4).

\section{Discusión}

Existen varios trabajos que abordan el problema de conservación de los peces del centro de México a nivel específico o regional (Lyons et al., 1998; Soto-Galera et al., 1998; Soto-Galera et al., 1999; Mercado-Silva et al., 2002; de la Vega-Salazar et al., 2003; Domínguez-Domínguez et al., 2007). Sin embargo, pocos trabajos se habían realizado con el objetivo de conocer de manera global el estado de conservación y las posibles causas de la desaparición de especies de un grupo completo de peces (DomínguezDomínguez et al., 2005b y Domínguez-Domínguez et al., 
Cuadro 2. Número de localidades y especies de goodeidos en distintas categorías de acuerdo a la región geográfica en la que habitan. Mesa Central, México

\begin{tabular}{|c|c|c|c|c|c|c|c|c|}
\hline \multirow[t]{2}{*}{ Región } & \multirow[t]{2}{*}{$\begin{array}{l}\text { Especies } \\
\text { endémicas }\end{array}$} & \multicolumn{3}{|c|}{$\begin{array}{c}\text { Núm. de localidades donde } \\
\text { se recolctaron especies } \\
\text { registradas }\end{array}$} & \multicolumn{3}{|c|}{$\begin{array}{l}\text { Núm. de especies } \\
\text { según su tolerancia* }\end{array}$} & \multirow[t]{2}{*}{$\begin{array}{l}\text { Especies } \\
\text { localmente } \\
\text { extintas }\end{array}$} \\
\hline & & $R$ & $A$ & $N$ & $S$ & $M$ & $T$ & \\
\hline Zacapu & 1 & 4 & 3 & 0 & 3 & 2 & 2 & 0 \\
\hline Chapala & 0 & 0 & 8 & 0 & & & & 4 \\
\hline Santiago & 0 & 1 & 4 & 20 & 2 & 2 & 3 & 3 \\
\hline Lerma bajo & 0 & 4 & 7 & 6 & 2 & 2 & 3 & 0 \\
\hline Lerma medio & 0 & 0 & 8 & 9 & 4 & 2 & 2 & 4 \\
\hline Lerma alto & 0 & 5 & 5 & 8 & 2 & 0 & 2 & 1 \\
\hline Zirahuén & 1 & 0 & 2 & 1 & 2 & 2 & 1 & 2 \\
\hline Pátzcuaro & 1 & 2 & 5 & 0 & 2 & 2 & 1 & 0 \\
\hline Balsas & 1 & 22 & 0 & 2 & 2 & 1 & 3 & 0 \\
\hline Cotija & 3 & 4 & 2 & 0 & 2 & 2 & 1 & 0 \\
\hline Ameca & 6 & 7 & 13 & 5 & 6 & 3 & 2 & 2 \\
\hline Cuitzeo & 0 & 4 & 9 & 4 & 4 & 2 & 2 & 1 \\
\hline $\begin{array}{l}\text { Armería } \\
\text { Ayuquila }\end{array}$ & 1 & 7 & 10 & 3 & 2 & 1 & 2 & 2 \\
\hline $\begin{array}{l}\text { Coahuayana } \\
\text { Tamazula }\end{array}$ & 2 & 6 & 8 & 0 & 3 & 1 & 1 & 1 \\
\hline Pánuco & 3 & 6 & 2 & 7 & 2 & 1 & 2 & 0 \\
\hline Valle de México & 1 & 0 & 2 & 6 & 1 & 0 & 0 & 0 \\
\hline Mezquital & 2 & 5 & 0 & 7 & $i ?$ & ¿? & ¿? & 0 \\
\hline $\begin{array}{l}\text { Purificación } \\
\text { Marabasco }\end{array}$ & 1 & 3 & 1 & 0 & 1 & 0 & 1 & 0 \\
\hline Total & 23 & 80 & 89 & 78 & -- & -- & -- & 20 \\
\hline
\end{tabular}

* De acuerdo a Lyons et al. (2000) y Mercado-Silva et al. (2002).

$R=$ donde todas las especies registradas fueron recolectadas; $A=$ donde al menos una de las especies registradas no fue recolectada; $N=$ donde ninguna de las especies registradas fue recolectada. $S=$ sensibles. $M=$ moderadamente sensibles. $T=$ tolerantes.

2006a). El presente estudio abarca el $95 \%$ de las zonas de distribución de la subfamilia Goodeinae y presenta un panorama general de la desaparición de poblaciones en cuencas del centro de México.

El alto número de extinciones locales registradas en la cuenca es dramático. En conjunto, un $68 \%$ del total de las localidades muestreadas registró al menos una especie de goodeido desaparecida. Por su parte, existen especies cuya distribución se ha reducido hasta un $40 \%$ en todas las regiones, llegando hasta un $100 \%$ la desaparición regional (v. gr. Santiago). A pesar de que esto puede verse únicamente como una reducción en el intervalo de distribución de una especie, el panorama para la conservación a largo plazo de los peces de esta familia es pesimista. Estudios previos han revelado que la mayoría de las especies de goodeidos presentan una fuerte diferenciación genética entre sus poblaciones (Doadrio y Domínguez, 2004), lo que deriva en una variación intraespecifica importante y divergencias poblacionales elevadas. Esto implica que cada población representa una parte de la variabilidad de toda la especie, y que dicha variabilidad podría estar representando adaptaciones evolutivas independientes, e incluso, en algunos casos, linajes evolutivos independientes (Domínguez-Domínguez et al., 2008). Por ello, debe considerarse que todas las poblaciones de una especie, en su conjunto, incrementan la adecuación de ésta. La fragmentación de hábitats y la extinción de poblaciones son quizás las principales causas de la pérdida de variabilidad genética en especies dentro de la subfamilia Goodeinae (Domínguez-Domínguez et al., 2007).

En cuanto a la resistencia de las especies a los cambios 
Cuadro 3. Tasa de permanencia de las especies de goodeidos por región; el valor representa la fracción del total de localidades donde aún prevalece la especie dentro de cada región

\begin{tabular}{|c|c|c|c|c|c|c|c|c|c|c|c|}
\hline Especies & $Z A C$ & CHA & $S A N$ & $L E A$ & $L E M$ & $L E B$ & $Z I R$ & $P A T$ & $B A L$ & $C O T$ & $A M E$ \\
\hline Allodontichthys hubbsi & & & & & & & & & & & \\
\hline Allodontichthys polylepis & & & & & & & & & & & 0.3 \\
\hline Allodontichthys tamazulae & & & & & & & & & & & \\
\hline Allodontichthys zonistius & & & & & & & & & & & \\
\hline Alloophorus robustus & 0.75 & 0 & 0 & & 0 & 0.5 & 0 & 1 & 1 & 1 & \\
\hline Allotoca catarinae & & & & & & & & & 1 & & \\
\hline Allotoca diazi & & & & & & & & 1 & & & \\
\hline Allotoca dugesii & & 0 & 0 & & 0.2 & 0.25 & 0.25 & 0.5 & & & \\
\hline Allotoca goslinae & & & & & & & & & & & 0.5 \\
\hline Allotoca maculata & & & & & & & & & & & 0.5 \\
\hline Allotoca meeki & & & & & & & 0.33 & & & & \\
\hline Allotoca zacapuensis & 1 & & & & & & & & & & \\
\hline Ataeniobius toweri & & & & & & & & & & & \\
\hline Ameca splendens & & & & & & & & & & & 0.5 \\
\hline Chapalichthys encaustus & & 1 & 0.33 & & & 0.75 & & & & & \\
\hline Chapalichthys pardalis & & & & & & & & & & 0.5 & \\
\hline Chapalichthys peraticus & & & & & & & & & & 1 & \\
\hline Characodon audax & & & & & & & & & & & \\
\hline Characodon garmani & & & & & & & & & & & \\
\hline Characodon lateralis & & & & & & & & & & & \\
\hline Girardinichthys multirradiatus & & & & 0.8 & & & & & 0.5 & & \\
\hline Chapalichthys encaustus & & 1 & 0.33 & & & 0.75 & & & & & \\
\hline Girardinichthys viviparus & & & & & & & & & & & \\
\hline Goodea atripinnis & 0.8 & 1 & 0.66 & 0.8 & 0.4 & 0.87 & 0.66 & 1 & & 1 & 0.75 \\
\hline Goodea gracilis & & & & & & & & & & & \\
\hline Hubbsina turneri & 1 & & & & 0 & & & & & & \\
\hline Ilyodon cortesae & & & & & & & & & 1 & & \\
\hline Ilyodon furcidens & & & & & & & & & & & 0.85 \\
\hline Ilyodon lennoni & & & & & & & & & ¿? & & \\
\hline Ilyodon whitei & & & & & & & & & 0.91 & & \\
\hline Neotoca bilineata & & & & & 0.33 & & & & & & \\
\hline Neoophorus regalis & & & & & & & & & & 0.5 & \\
\hline Skiffia francesae & & & & & & & & & & & 0 \\
\hline Skiffia lermae & 1 & & & 0.5 & 0 & & 0 & 0.5 & & & \\
\hline Skiffia multipunctata & & 0 & 0 & & & 0.5 & & & & & \\
\hline Xenoophorus captivus & & & & & & & & & & & \\
\hline Xenotoca eiseni & & & 0.15 & & & & & & & & 0.5 \\
\hline Xenotoca melanosoma & & 0 & & & & & & & & & 0.5 \\
\hline Xenotaenia resolanae & & & & & & & & & & & \\
\hline Xenotoca variata & 1 & 0.33 & 0.25 & 0 & 0.75 & 0.5 & & & & & \\
\hline Zoogoneticus quitzeoensis & 1 & & & & 0 & 0.5 & & & & & 0.33 \\
\hline Zoogoneticus tequila & & & & & & & & & & & 0 \\
\hline Total previos & 7 & 7 & 7 & 4 & 8 & 7 & 5 & 5 & 6 & 5 & 12 \\
\hline Total actual & 7 & 4 & 4 & 3 & 4 & 7 & 3 & 5 & 6 & 4 & 10 \\
\hline Promedio TP & 0.9357 & 0.3328 & 0.1985 & 0.525 & 0.21 & 0.5528 & 0.248 & 0.8 & 0.882 & 0.8 & 0.432 \\
\hline
\end{tabular}


Cuadro 3. Continúa

\begin{tabular}{|c|c|c|c|c|c|c|c|c|c|c|}
\hline Especies & $C U I$ & $A Y U$ & $C U T$ & $P A N$ & $V A M$ & $M E Z$ & $P U M$ & $\begin{array}{l}\text { Total } \\
\text { previos }\end{array}$ & $\begin{array}{l}\text { Total } \\
\text { actual }\end{array}$ & $\begin{array}{l}\text { Valor de } \\
\text { presencia }\end{array}$ \\
\hline Allodontichthys hubbsi & & & 0.33 & & & & & 1 & 1 & 0.33 \\
\hline Allodontichthys polylepis & & & & & & & & 1 & 1 & 0.33 \\
\hline Allodontichthys tamazulae & & & 0.45 & & & & & 1 & 1 & 0.45 \\
\hline Allodontichthys zonistius & & 0.7 & & & & & & 1 & 1 & 0.7 \\
\hline Alloophorus robustus & 0.5 & & & & & & & 10 & 6 & 0.475 \\
\hline Allotoca catarinae & & & & & & & & 1 & 1 & 1 \\
\hline Allotoca diazi & & & & & & & & 1 & 1 & 1 \\
\hline Allotoca dugesii & 0.33 & & & & & & & 7 & 5 & 0.218 \\
\hline Allotoca goslinae & & & & & & & & 1 & 1 & 0.5 \\
\hline Allotoca maculata & & & & & & & & 1 & 1 & 0.5 \\
\hline Allotoca meeki & & & & & & & & 1 & 1 & 0.33 \\
\hline Allotoca zacapuensis & & & & & & & & 1 & 1 & 1 \\
\hline Ataeniobius toweri & & & & 0.33 & & & & 1 & 1 & 0.33 \\
\hline Ameca splendens & & & & & & & & 1 & 1 & 0.5 \\
\hline Chapalichthys encaustus & & & & & & & & 3 & 3 & 0.693 \\
\hline Chapalichthys pardalis & & & & & & & & 1 & 1 & 0.5 \\
\hline Chapalichthys peraticus & & & & & & & & 1 & 1 & 1 \\
\hline Characodon audax & & & & & & 0.5 & & 1 & 1 & 0.5 \\
\hline Characodon garmani & & & & & & & & 1 & 0 & 0 \\
\hline Characodon lateralis & & & & & & 0.33 & & 1 & 1 & 0.33 \\
\hline Girardinichthys multirradiatus & & & & & & & & 2 & 2 & 0.65 \\
\hline Chapalichthys encaustus & & & & & & & & 3 & 3 & 0.693 \\
\hline Girardinichthys viviparus & & & & ¿? & 0.33 & & & 2 & 1 & 0.33 \\
\hline Goodea atripinnis & 1 & 0.8 & & & & & & 12 & 12 & 0.811 \\
\hline Goodea gracilis & & & & 0.66 & & & & 1 & 1 & 0.66 \\
\hline Hubbsina turneri & 0 & & & & & & & 3 & 1 & 0.333 \\
\hline Ilyodon cortesae & & & & & & & & 1 & 1 & 1 \\
\hline Ilyodon furcidens & & 0.75 & & & & & 1 & 3 & 3 & 0.866 \\
\hline Ilyodon lennoni & & & & & & & & 1 & 1 & \\
\hline Ilyodon whitei & & & 0.8 & & & & & 2 & 2 & 0.855 \\
\hline Neotoca bilineata & 0.5 & & & & & & & 2 & 2 & 0.415 \\
\hline Neoophorus regalis & & & & & & & & 1 & 1 & 0.5 \\
\hline Skiffia francesae & & & & & & & & 1 & 0 & 0 \\
\hline Skiffia lermae & 0.75 & & & & & & & 6 & 3 & 0.458 \\
\hline Skiffia multipunctata & & & & & & & & 3 & 1 & 0.166 \\
\hline Xenoophorus captivus & & & & 0.33 & & & & 1 & 1 & 0.33 \\
\hline Xenotoca eiseni & & & 0.33 & & & & & 3 & 3 & 0.326 \\
\hline Xenotoca melanosoma & & 0 & 0 & & & & & 4 & 1 & 0.125 \\
\hline Xenotaenia resolanae & & & & & & & 0.5 & 1 & 1 & 0.5 \\
\hline Xenotoca variata & 0.75 & & & 0.75 & & & & 8 & 8 & 0.541 \\
\hline Zoogoneticus quitzeoensis & 0.75 & 0 & & & & & & 6 & 4 & 0.43 \\
\hline Zoogoneticus tequila & & & & & & & & 1 & 0 & 0 \\
\hline Total previos & 8 & 5 & 5 & 5 & 1 & 2 & 2 & & & \\
\hline Total actual & 7 & 3 & 4 & 4 & 1 & 2 & 2 & & & \\
\hline Promedio TP & 0.572 & 0.45 & 0.382 & 0.517 & 0.33 & 0.415 & 0.75 & 0.518 & & 0.499 \\
\hline
\end{tabular}

Total previos, total de regiones donde se ha registrado la presencia de la especie; Total actual, total de regiones donde aún prevalece la especie por región. ZAC, Zacapu; CHA, Chapala; $S A N$, Santiago y Verde; LEA, Lerma Alto; LEM, Lerma Medio; LEB, Lerma Bajo, ZIR, Zirahuén; PAT, Pátzcuaro; BAL, Balsas; $C O T$, Cotija; $A M E$, Ameca; $C U I$, Cuitzeo; $A Y U$, Ayuquila. Armería; CUT, CoahuayanaTamazula; PAN, Pánuco; VAM, Valle de México; MEZ, Mezquital; PUM, Purificación-Mascota. 


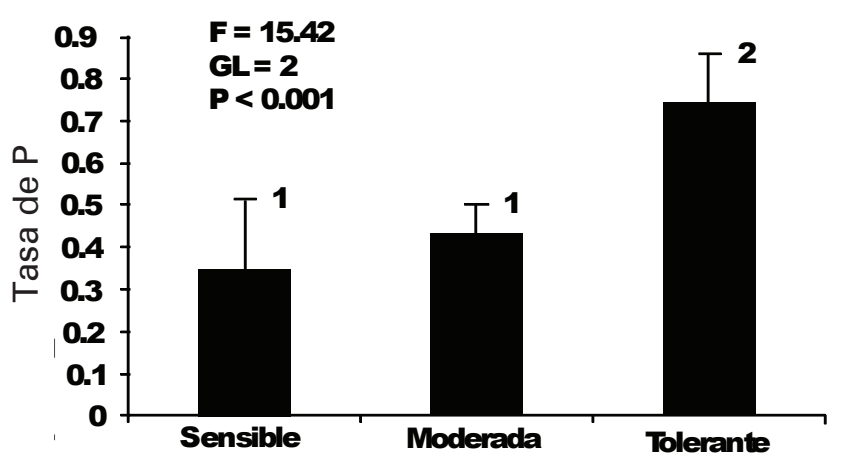

Figura 2. Tasa de permanencia (TP) promedio con la desviación estándar de cada grupo de especies de goodeidos clasificado por su vulnerabilidad de acuerdo con Lyons et al. (2000) y MercadoSilva et al. (2002). Los números arriba de cada barra indican diferencias significativas en el análisis post-hoc.

Cuadro 4. Clasificación de las 18 regiones (centro de México) por la tasa de permanencia (TP) promedio de las especies que contienen

\begin{tabular}{|c|c|}
\hline Grupo & Cuenca (TP promedio) \\
\hline 1 & $\begin{array}{l}\text { Santiago (0.19); Lerma medio (0.21); Zirahuén } \\
(0.24) \text {; Valle de México }(0.33) \text {; Chapala }(0.33) \text {. }\end{array}$ \\
\hline 2 & $\begin{array}{l}\text { Coahuayana-Tamazula (0.38); Mezquital (0.42); } \\
\text { Ameca (0.43); Ayuquila-Armería (0.45); Pánuco } \\
(0.52) \text {; Lerma alto (0.53); Lerma bajo (0.55); } \\
\text { Cuitzeo }(0.57) \text {. }\end{array}$ \\
\hline 3 & $\begin{array}{l}\text { Purificación-Marabasco (0.75); Pátzcuaro (0.80); } \\
\text { Cotija (0.80); Balsas (0.88); Zacapu (0.93). }\end{array}$ \\
\hline
\end{tabular}

$1=$ regiones con especies con TP promedio menor al 33\%; $2=$ regiones con especies con TP promedio entre el 33 y el $66 \% ; 3=$ regiones en las que la permanencia ha sido mayor al $66 \%$.

del hábitat, la desaparición de aquellas más sensibles en un mayor número de localidades, en comparación con la poca reducción de las tolerantes, sugiere que la clasificación realizada por Lyons et al. (2000) y Mercado-Silva et al. (2002), es acertada y coincide con estudios realizados por Mercado-Silva et al. (2006), lo que refuerza la idea de que existen diferencias en la capacidad de resistencia de las especies a los cambios ambientales, por lo que las políticas de conservación deben ser diseñadas de manera particular para cada grupo de organismos, e incluso para cada población. De igual forma, las especies que tienen un mayor número de extinciones locales están representadas por especies carnívoras (v.gr. Alloophorus robustus y Allotoca dugesii), como se corrobora en los estudios de Soto-Galera et al. (1999) y Mercado-Silva et al. (2006), quienes registraron una disminución significativa de

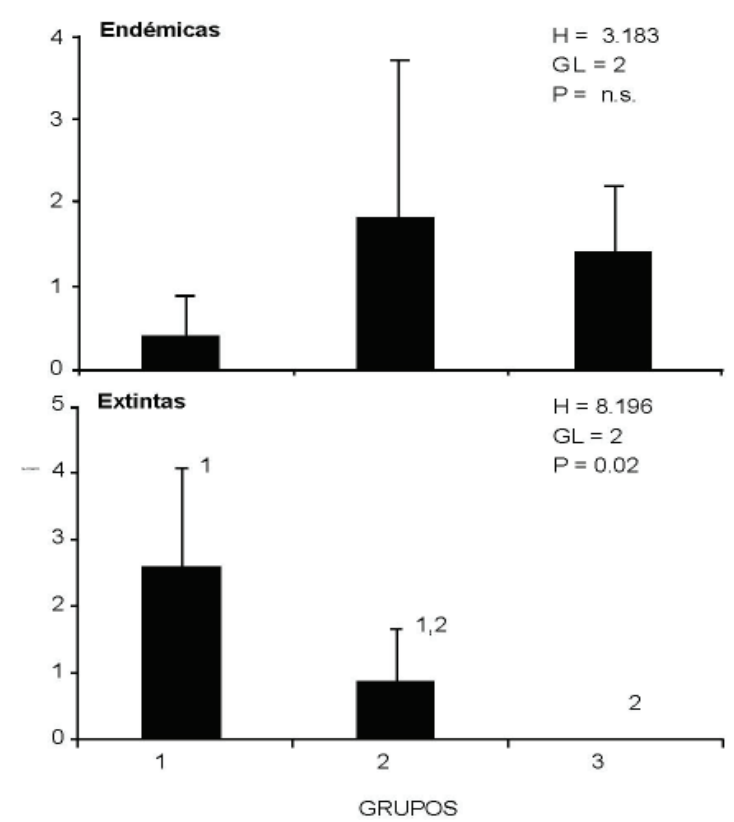

Figura 3. Número promedio de especies endémicas y extintas con la desviación estándar que presenta cada tipo de región $(1,2,3)$ de acuerdo con la clasificación por la TP promedio. Los números arriba de cada barra indican diferencias significativas en el análisis post-hoc.

especies carnívoras en la cuenca del río Morelia y río Laja asociada a cambios en la cadena trófica o destrucción del hábitat de las presas. Las especies que se encuentran encima de la cadena trófica (los carnívoros) por lo general son los primeros en desaparecer puesto que dependen de una estructura sólida en la pirámide alimentaria.

El mayor número de especies de goodeidos habitan en las zonas altas de las cuencas, excepto las especies de los géneros Ilyodon, Allodontichthys, y las especies Xenotaenia melanosoma y $X$. eiseni), las cuales a su vez son habitantes de las áreas más perturbadas por la acción del hombre (v.gr. el río Ameca). Comparando las localidades que están incluidas en el grupo 1 (regiones con especies que en promedio tuvieron una tasa de permanencia igual o menor al 33\%), se demuestra que dichas áreas son las más perturbadas (v.gr. Chapala y Valle de México), mientras que algunas de las localidades del grupo 3 presentan un menor grado de alteraciones (v.gr. Balsas) (Lyons et al., 1998; Soto-Galera et al., 1999; Mercado-Silva et al., 2002; Domínguez-Domínguez et al., 2006a). De la misma forma, cuencas con un alto número de endemismos (v.gr. Ameca con 11 especies de goodeidos, 6 de ellas endémicas) presentan un mayor número de localidades donde ha desaparecido al menos una de la especies con registros previos. Las localidades más perturbadas coinciden con el número de especies extintas localmente, y es importante 


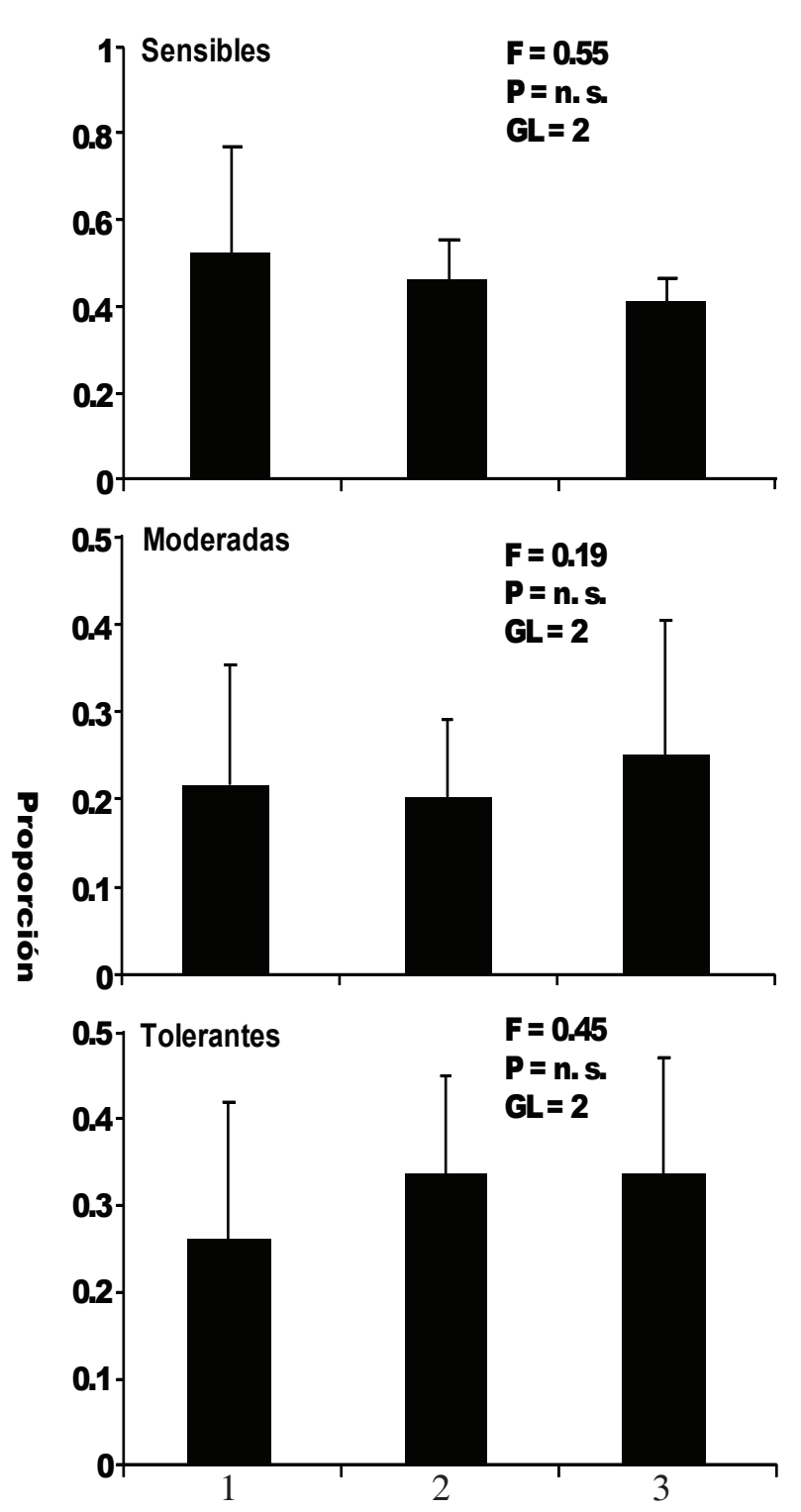

Figura 4. Número promedio de especies sensibles, moderadas y tolerantes con desviación estándar que presenta cada tipo de región $(1,2,3)$ de acuerdo con la clasificación por la TP promedio. $1=$ con especies con promedio menor al $33 \% ; 2=$ con especies con promedio entre el 33 y el $66 \% ; 3=$ especies con permanencia mayor al $66 \%$.

hacer notar que varias de estas localidades representan las zonas de mayor endemismo de goodeidos.

Lo anterior coincide con la falta de diferencias significativas en relación a la proporción de especies sensibles por cada región (Fig. 4). Por lo tanto, si el grupo 1 cuenta con una tasa mayor de extinción de especies, pero con la misma proporción de especies sensibles que el grupo 2 y 3 , podría suponerse que la desaparición local de poblaciones se deba claramente a una fuerte perturbación humana. Esto difiere con lo encontrado por Soto-Galera et al. (1998; 1999) y Mercado-Silva et al. (2006), quienes demostraron una mayor desaparición de especies de peces sensibles en regiones particulares (los ríos Lerma, Morelia y Laja). Las diferencias podrían estar relacionadas con el tipo de regiones incluidas en este trabajo (v. gr. Balsas y Pátzcuaro) que han sido menos perturbadas que el río Lerma o río Morelia. Otra posibilidad es que en el presente trabajo sólo se utlizó un grupo de peces (goodeidos) a diferencia del trabajo previo que utilizó la comunidad completa de peces. La diferencia sugiere que estos datos tendrían que tomarse con cautela al momento de ser extrapolados. Este trabajo representa una perspectiva de la problemática a la que se enfrenta un grupo particular de peces, los goodeidos, por lo que es necesario el planteamiento de estudios más detallados que incluyan más grupos de peces y a una escala local dentro del centro de México.

Los goodeidos del centro de México están considerados en el mundo como un grupo de peces con alto riesgo de extinción (Duncan y Lockwood, 2001). Por ello, este trabajo confirma y pone de manifiesto la urgente necesidad de desarrollar programas de restauración ecológica y manejo de áreas clave para la conservación que reviertan esta tendencia de extinciones locales. En las visitas a las localidades se pudo observar que los cuerpos de agua alimentados por manantiales o los arroyos de alta montaña son los que conservan las especies que estaban registradas en las diferentes localidades. Por ello, dichas zonas con poca perturbación humana o manantiales con una alta capacidad de amortiguamiento a los contaminantes, debido al flujo continuo de agua, están actuando como refugio de especies sensibles (v.gr. La Mintzita, La Luz, Orandino, Zacapu en el estado de Michoacán, Guadalupe Aguilera, Los Berros, El Toboso, en el estado de Durango, La Media Luna y Arroyo Jesús María en San Luis Potosí).

\section{Agradecimientos}

A todas las personas que colaboraron en las jornadas de campo y laboratorio, en especial a Asdrubal Molina, Karen Arias, Marisol Simón, Adolfo de Sostoa, Hugo Mejía, Rogelio Rosas, Jen Nightingale, Jean de Moree, Ludo Couvreur, Kees de Jong, Juan Miguel Artigaz, Arthur Frizby, Juan Carlos Merino, Nancy Calderón, Xavier Madrigal, Nayeli Gurrola y Adrián Pompa. El financiamiento parcial para este proyecto fue otorgado por el Consejo Estatal de Ciencia y Tecnología-Michoacán y el Zoológico de Chester del Reino Unido. Un especial agradecimiento a Ivan Dibble por su apoyo a través del Hobbyist Aqualab Conservation Project (HALCP) el 
cual recibe fondos de organizaciones de acuaristas de Europa y de Estados Unidos de América. ODD, LHEV y RPR agradecen la beca de posgrado recibida del Consejo Nacional de Ciencia y Tecnología. Parte de este estudio se llevó a cabo gracias a los recursos otorgados a G.P.P.L. de los programas PAPIIT-UNAM IN220605 y CONACyT 47233.

\section{Literatura citada}

Álvarez del Villlar, J. 1970. Peces mexicanos (claves). Secretaría de Industria y Comercio, Instituto Nacional de Investigaciones Biológico-Pesqueras y Comisión Nacional de Pesca, México, D.F. 166 p.

Athie, M. 1987. Calidad y cantidad del agua en México. Fundación Universo Veintiuno, México, D.F. 192 p.

Barbour, C. D. 1973. A biogeographical history of Chirostoma (Pisces:Aterinidae): a species flock from the Mexican plateau. Copeia 3:533-556.

Cotler, H., M. Mazari-Hiriart y J. de Anda Sánchez (eds.) Atlas de la cuenca Lerma-Chapala. Construyendo una visión conjunta. p. 196.

Contreras-Balderas, S., P. Almada-Villela, M. de L. LozanoVilano y M. García-Ramírez. 2003. Freshwater fish at risk or extinct in México, A checklist and review. Reviews in Fish Biology and Fisheries 12:241-251.

De la Vega-Salazar, M. Y., E. Ávila-Luna y C. Macías-García. 2003. Ecological evaluation of local extinction: the case of two genera of endemic Mexican fish, Zoogoneticus and Skiffia. Biodiversity and Conservation 12: 2043-2056.

Doadrio, I. y O. Domínguez. 2004. Phylogenetic relationship within the fish family Goodeidae based on Cytochrome b sequence data. Molecular Phylogenetics and Evolution 31:416-430.

DOF (Diario Oficial de la Federación). 2002. Norma Oficial Mexicana de Ecología 059-2001. Secretaría del Medio Ambiente y Recursos Naturales, Dirección General de Regulación Ambiental, Gobierno de la República, México, D.F. 6 de marzo.

Domínguez-Domínguez, O., N. Mercado-Silva y J. Lyons. 2005a. Conservation status of Mexican goodeids: problems, perspectives, and solutions. In Proceedings of the II International Symposium on Livebearing Fishes, M. C. Uribe-Aranzabal y H. Grier (eds.). New Life, Homestead, Florida. p. 495-504.

Domínguez-Domínguez, O., N. Mercado-Silva, J. Lyons y H. J. Grier. 2005b. Goodeid fishes photos. In Proceedings of the II International Symposium on Livebearing Fishes, M. C. Uribe-Aranzabal y H. Grier (eds.) New Life, Homestead, Florida. p. 505-549.

Domínguez-Domínguez, O., E. Martínez-Meyer, L. Zambrano y G. Pérez-Ponce de León. 2006a Using ecological-niche modeling to prioritize conservation areas in the Mesa Central of Mexico: a case study with livebearing freshwater fishes (Goodeidae). Conservation Biology 20:1730-1739.

Domínguez-Domínguez O., I. Doadrio y G. Pérez-Ponce de
León. 2006b. Historical biogeography of some river basins in Central Mexico evidenced by their goodeine freshwater fishes: a preliminary hypothesis using secondary brooks parsimony analysis (BPA). Journal of Biogeography 33:1437-1447.

Domínguez-Domínguez, O., L. Boto, F. Alda, G. Pérez-Ponce de León e I. Doadrio, 2007. Human impacts on basins of the Mesa Central of Mexico, and its genetic effects on an endangered fish, Zoogoneticus quitzeoensis. Conservation Biology 21:168-180.

Domínguez-Domínguez, O., F. Alda, G. Pérez-Ponce de León, J. L. García-Garitagoitia e I. Doadrio. 2008. Evolutionary history of the endangered fish Zoogoneticus quitzeoensis (Beab, 1898) (Cyprinodontiformes: Goodeidae) using a sequential approach to phylogeography based on mitochondrial and nuclear DNA data. Evolutionary Biology. BMC Evolutionary Biology 8:161.

Duncan, J. R. y J. L. Lockwood. 2001. Extintion in a field of bullets: a search for causes in the decline of the world's freshwater fishes. Biological Conservation 102:97-105.

Echelle, A. A. y A. F. Echelle. 1984. Evolutionary generics of a "species flock": Aterinid fishes on the Mesa Central of Mexico. In Evolution of fish species flocks, A. A. Echelle y I. Kornfield (eds.). University of Maine at Orono Press. p. 93-110.

Efford, I. E., C. Macías-García y J. D. Williams. 1997. Facing the challenges of invasive alien species in North America. Global Biodiversity 7:25-30.

Escalera-Vázquez, L. H. 2006. Influencia de Poecilia mexicana (especie introducida) en el desplazamiento competitivo de Zoogoneticus tequila, Ameca splendens y Goodea atripinnis (especies nativas) de Teuchitlán, Jalisco. Tesis maestría, Posgrado en Ciencias Biológicas, Instituto de Biología, UNAM. $61 \mathrm{p}$.

Ledesma-Ayala, C. L. 1987. Estudio ictiológico del Río Duero, Michoacán. Tesis, Universidad Autónoma de Nuevo León, Monterrey. 89 p.

Lyons, J., S. Navarro-Pérez, P. A. Cochran, E. C. Santana y M. Guzmán-Arroyo. 1995. An index of biotic integrity based on fish assemblages for the conservation of streams and rivers in west-central Mexico. Conservation Biology 9:569-584.

Lyons, J., G. González-Hernández, E. Soto-Galera y M. GuzmánArroyo. 1998. Decline of freshwater fishes and fisheries in selected drainages of west-central México. Fisheries Management, American Fisheries Society 23:10-18.

Lyons, J., A. Gutiérrez-Hernández, E. Díaz-Pardo, E. SotoGalera, M. Medina-Nava y R. Pineda-López. 2000. Development of a preliminary index of biotic integrity based on fish assemblages to assess ecosystem condition in the lakes of central Mexico. Hydrobiologia 418:57-72.

Lytle, D. A. y P. N. LeRoy. 2004. Adaptation to natural flow regimes. Trends in Ecology and Evolution 19:94-100.

Maezono, Y. y Miyashita T. 2003. Community-level impacts induced by introduced largemouth bass and bluegill in farm ponds in Japan. Biological Conservation 109:111-121.

Matthews, W. J. y E. Marsh-Matthews. 2003. Effects of drought on fish across axes and ecological complexity. Freshwater Biology 48:1232-1253. 
Miller, R. R., W. L. Minckley y S. M. Norris. 2005. Freshwaters fishes of Mexico. University of Chicago Press. 490 p.

Mercado-Silva, N., J. Lyons, G. Salgado y M. Medina-Nava. 2002. Validation of a fish-based index of biotic integrity for streams and rivers of central Mexico. Reviews in Fish Biology and Fisheries 12:179-191.

Mercado-Silva, N., J. Lyons, E. Díaz-Pardo, A. GuitiérrezHernández, C. P. Ornelas-García, C. Pedraza-Lara y V. J. V. Zanden. 2006. Long-term changes in the fish assemblage of the Laja River, Guanajuato, central Mexico Aquatic Conservation: Marine and Freshwater Ecosystems 16:533546.

Siegel, S. y N. J. Castellan. 1988. Nonparetric statistics for the behavioural sciences. Mc Graw Hill, New York. 399 p.

Soto-Galera, E., E. E. Díaz-Pardo, E. López-López y J. Lyons.
1998. Fish as indicators of environmental quality in the Río Lerma Basin, México. Aquatic Ecosystem Health and Management 1:267-276.

Soto-Galera, E., J. Paulo-Maya, E. López-Lópeza, J. A. SernaHernández y J. Lyons. 1999. Change in fish fauna as indication of aquatic ecosystem condition in Río Grande de Morelia-Lago de Cuitzeo basin, Mexico. Environmental Management 24:133-140.

Weis, J. S., G. M. Smith y T. Zhou. 1999. Altered predator/prey behaviour in polluted environments: implications for fish conservation. Environmental Biology of Fishes 55:43-51.

Zambrano, L., Perrow, M. Aguirre-Hidalgo, V. y Macías-García, C. 1999. Impact of introduced carp (Cyprinus carpio) in subtropical shallow ponds in central Mexico. Journal of Aquatic Stress Ecosystems and Recovery 6:281-288. 